\title{
Risco nutricional em pacientes adultos e idosos de um hospital do sul do Brasil
}

\author{
Nutritional risk in elderly and adult patients of a hospital in Southern Brazil
}

\author{
Renata Araujo Rodrigues Gabbardoํㅜ, Camile Boscaini ${ }^{2}$
}

${ }^{1}$ Graduanda em Nutrição pela Faculdade Cenecista de Bento Gonçalves, Bento Gonçalves, RS; ${ }^{2}$ Nutricionista, Mestre em Ciências da Saúde pela Universidade Federal de Ciências da Saúde de Porto Alegre, Porto Alegre, RS.

\section{RESUMO}

Objetivos: Verificar a prevalência do risco nutricional na admissão hospitalar, utilizando o protocolo Nutrition Risk Screening (NRS 2002) e identificar quais foram os aspectos desse protocolo que mais contribuiram para o escore final.

Métodos: Foi realizado um estudo transversal com pacientes adultos e idosos de ambos os sexos, admitidos nos departamentos de internação clínica (exceto maternidade, emergência e unidade de saúde mental) de um hospital da cidade de Farroupilha, Rio Grande do Sul. Os indivíduos foram avaliados através do protocolo NRS 2002, nos primeiros três dias após admissão hospitalar.

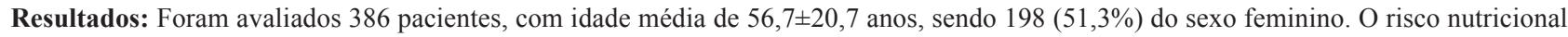
esteve presente em $57(14,8 \%)$ dos pacientes. Na primeira parte da triagem nutricional, $315(81,6 \%)$ pacientes tiveram pelo menos uma resposta positiva indicando risco nutricional, sendo a redução de ingestão alimentar na última semana a mais prevalente. Na segunda parte observou-se que em relação ao estado nutricional, a proporção do estado grave esteve presente em 21 pacientes (36,8\% da amostra em risco). Quanto à gravidade da doença, prevaleceu o fator de risco leve em 29 (50,9\%) pacientes. A pontuação adicional, através da pontuação da variável idade acima de 70 anos, contribuiu para caracterizar o risco nutricional em 35 pacientes $(61,4 \%)$.

Conclusões: Observou-se elevada proporção de pacientes em risco nutricional, sendo a redução na ingestão alimentar na última semana a variável que mais contribuiu para dar sequência à segunda etapa do processo de triagem nutricional.

DESCRITORES: AVALIAÇÃO NUTRICIONAL; INGESTÃO DE ALIMENTOS; ASSISTÊNCIA AO PACIENTE.

\section{ABSTRACT}

Aims: To determine the prevalence of nutritional risk at admission using the Nutrition Risk Screening (NRS 2002) protocol, and to identify which aspects of this protocol contributed most to the final score.

Methods: A cross-sectional study included adult and elderly patients of both sexes, admitted to clinical departments (except maternity, emergency and mental health unit) of a hospital in the city of Farroupilha, Rio Grande do Sul state, Brazil. Individuals were assessed through NRS 2002 in the first three days after admission.

Results: A total of 386 patients were evaluated. They had a mean age of $56.7 \pm 20.7$ years, and 198 (51.3\%) were female. Nutritional risk was present in $57(14.8 \%)$ patients. In the first part of the nutritional screening, $315(81.6 \%)$ patients had at least one positive response indicating nutritional risk, being the reduction of food intake in the last week the most prevalent. In the second part it was noted that, in relation to nutritional status, the proportion of the severe condition was present in 21 patients (36.8\% of the sample at risk). In relation to severity of disease, the prevailing condition was mild disease, in 29 (50.9\%) patients. Additional score given by age over 70 years helped to characterize nutritional risk in 35 patients $(61.4 \%)$.

Conclusions: There was a high proportion of patients with nutritional risk. Reduction in food intake in the previous week was the variable that contributed most to give sequence to the second stage of the nutrition screening process.

KEY WORDS: NUTRITION ASSESSMENT; FOOD INTAKE; PATIENT CARE. 


\section{INTRODUÇÃO}

A desnutrição no ambiente hospitalar tem crescido significativamente em todo o mundo, sendo que sua prevalência varia de $20 \%$ a $50 \%$ em diferentes estudos. ${ }^{1}$ No Brasil, 48\% dos pacientes internados na rede pública de saúde apresentam algum grau de desnutrição, podendo variar a prevalência conforme a região estudada. Alguns pacientes já são admitidos no hospital com desnutrição e outros a desenvolvem após a internação. ${ }^{2}$

Mesmo sendo descrita como altamente prevalente ao longo dos anos, a desnutrição continua a ser pouco reconhecida pela equipe de saúde e, ainda nos dias atuais, frequentemente não é diagnosticada. A desnutrição pode complicar a evolução clínica do paciente, contribuindo para o aumento da morbimortalidade, tempo de internação e despesas hospitalares. ${ }^{1,3-6}$ A avaliação nutricional é um processo sistemático, sendo o primeiro passo da assistência nutricional. É fundamental que a primeira avaliação seja realizada imediatamente após a internação, com o objetivo de reduzir possíveis complicações relacionadas à desnutrição. ${ }^{7,8}$

O risco nutricional de pacientes hospitalizados é avaliado pela combinação do estado nutricional atual com a gravidade da doença, sendo que o primeiro é composto pelas variáveis índice de massa corporal (IMC), perda de peso recente e ingestão dietética durante a última semana antes da admissão. ${ }^{9} \mathrm{~A}$ Triagem de Risco Nutricional (NRS 2002 - Nutrition Risk Screening 2002) ${ }^{10}$ é um instrumento para triagem nutricional que foi revisado e apoiado pela European Society for Parenteral and Enteral Nutrition (ESPEN) e, quando comparada com outras ferramentas de triagem, mostrou-se superior para identificar o estado nutricional de pacientes adultos hospitalizados. A avaliação independe do tipo de doença e da idade do paciente, além de não ter custo adicional ao serviço e poder ser efetuada por nutricionistas, enfermeiros ou médicos. ${ }^{11-13} \mathrm{~A}$ triagem nutricional no âmbito hospitalar permite detectar os pacientes em risco nutricional, e, dessa forma, realizar intervenção nutricional primária, permitindo priorizar e agilizar o atendimento aos pacientes, e assim, através de medidas preventivas, evitar que a situação se agrave devido às rotinas hospitalares adversas que podem levar à ingestão insuficiente de nutrientes. ${ }^{14-17}$

O objetivo deste estudo foi verificar a prevalência do risco nutricional na admissão hospitalar em pacientes avaliados pelo protocolo NRS 2002 e quais foram os aspectos deste que mais contribuíram para a pontuação final do score, em um hospital do sul do Brasil.

\section{MÉTODOS}

Foi realizado um estudo transversal, com pacientes adultos e idosos hospitalizados nas unidades de internação clínica, no período de maio a julho de 2013, em um hospital geral localizado na cidade de Farroupilha, estado do Rio Grande do Sul, o qual atende $60 \%$ de pacientes SUS e $40 \%$ pacientes conveniados ou particulares.

Foram incluídos no estudo indivíduos adultos (20 a 59 anos) e idosos (>60 anos) de ambos os sexos e diferentes etnias, hospitalizados em diversas unidades do hospital, independentemente do motivo da internação, que estavam lúcidos, orientados e conscientes, conforme avaliação da equipe de enfermagem, e que aceitaram participar do estudo através da assinatura do termo de consentimento livre e esclarecido, o qual pôde ser assinado pelo paciente ou responsável. Foram excluídos os pacientes internados no pronto socorro, na maternidade e na unidade de saúde mental.

Com auxílio do recurso Statcalc do programa Epi Info, o cálculo do tamanho da amostra foi baseado em estimativas provenientes de uma pesquisa multicêntrica em hospitais de rede pública do país, atingindo 12 estados e o Distrito Federal, envolvendo 4000 pacientes internados, observando prevalência de $48,1 \%$ de desnutrição hospitalar ${ }^{2}$ com um nível de confiança de $95 \%$ e margem de erro de $5 \%$, sendo estimada uma amostra de 384 pacientes. Considerando as possíveis perdas, esse número foi acrescido de $25 \%$, totalizando 479 indivíduos hospitalizados. Assim, foram selecionados inicialmente 479 pacientes consecutivos dentro do período de estudo, que atendiam aos critérios de inclusão mencionados. Entre estes, 93 pacientes não completaram todos os passos para a avaliação da triagem nutricional, sendo excluídos do banco de dados, resultando em uma amostra de 386 pacientes, que atendeu ao cálculo de tamanho amostral estimado.

Para a coleta de dados foi utilizada a ferramenta NRS $2002^{10}$ em que a avaliação acontece em duas etapas: a primeira é composta por quatro perguntas, referentes ao IMC inferior a 20,5, perda de peso nos três meses anteriores, redução da ingestão alimentar na semana anterior e presença de doença grave, mau estado geral ou internação em unidade de tratamento intensivo (UTI). Se uma das respostas for positiva, a próxima etapa é iniciada, e o estado nutricional e a gravidade da doença do paciente são pontuados, juntamente com a idade. Se a idade for igual ou maior que 70 anos, um ponto é adicionado e são realizadas 
medidas antropométricas (peso e altura) e calculado o IMC. A NRS 2002 tem os seguintes pontos de corte: Escore total $\geq 3$ : o paciente está em risco nutricional e o cuidado nutricional deve ser iniciado. Escore total $<$ 3: reavaliar o paciente semanalmente (Quadro 1). ${ }^{10}$

Os pacientes foram avaliados no leito em que permaneciam internados. Nos pacientes em que foi possível realizar a antropometria, os equipamentos utilizados foram balança portátil e estadiômetro portátil, ambos da marca Wiso (Florianópolis, SC). Para a medida do peso corporal os indivíduos foram orientados a usar roupas leves e permanecer com postura ereta na balança. $\mathrm{O}$ mesmo procedimento foi repetido três vezes pelo mesmo entrevistador e o registro do peso foi a média das três aferições. A estatura foi mensurada através do estadiômetro portátil. Foi solicitado ao entrevistado que, descalço, encostasse a cabeça no equipamento e mantivesse a parte posterior dos joelhos e parte posterior dos pés bem unidos. O mesmo procedimento foi repetido três vezes pelo mesmo entrevistador e o registro da estatura foi a média das três aferições. O IMC foi calculado pela divisão do peso (em quilos) pelo quadrado da altura (em metros). Para os pacientes em que não foi possível a aferição do peso e da estatura, por não conseguirem sair do leito, as medidas foram referidas pelo próprio paciente e/ou por familiares, conforme as instruções da NRS 2002. ${ }^{10}$

As outras variáveis analisadas (gênero, idade, doença de base e tempo de internação) foram obtidas através dos registros do prontuário do paciente, seguindo orientações declaradas no Termo de Confidencialidade para utilização do banco de dados.

O projeto foi aprovado pelo Comitê de Ética e Pesquisa da Faculdade Cenecista de Bento Gonçalves pelo parecer de número 260712 na data de 04 de abril de 2013.

Os dados foram organizados no programa Microsoft Excel $^{\circledR}$. As variáveis quantitativas foram descritas por média e desvio padrão e as categóricas por frequências absolutas e relativas. Para avaliar a associação entre as variáveis, os testes qui-quadrado de Pearson ou exato de Fisher foram aplicados. O nível de significância adotado foi de $5 \%(\mathrm{p} \leq 0,05)$ e as análises foram realizadas no programa SPSS versão 18.0 .

Quadro 1. Segunda etapa da Triagem de Risco Nutricional NRS 2002 (Nutritional Risk Screening) - conforme Kondrup et al. ${ }^{10}$

\begin{tabular}{|c|c|c|c|}
\hline \multicolumn{2}{|r|}{ Estado nutricional } & \multicolumn{2}{|c|}{$\begin{array}{l}\text { Gravidade da doença } \\
\text { (efeito do estresse metabólico no aumento das } \\
\text { necessidades nutricionais) }\end{array}$} \\
\hline $\begin{array}{l}\text { Ausente } \\
\text { (Pontuação 0) }\end{array}$ & Estado nutricional normal. & $\begin{array}{l}\text { Ausente } \\
\text { (Pontuação 0) }\end{array}$ & Necessidades nutricionais normais. \\
\hline $\begin{array}{l}\text { Leve } \\
\text { (Pontuação 1) }\end{array}$ & $\begin{array}{l}\text { Perda de peso }>5 \% \text { em } 3 \text { meses ou; } \\
\text { Ingestão alimentar abaixo de } 50-75 \% \text { da } \\
\text { necessidade normal na semana anterior. }\end{array}$ & $\begin{array}{l}\text { Leve } \\
\text { (Pontuação 1) }\end{array}$ & $\begin{array}{l}\text { Fratura de quadril; } \\
\text { pacientes crônicos com complicações agudas: } \\
\text { cirrose, doença pulmonar obstrutiva crônica } \\
\text { (DPOC); } \\
\text { diabetes, câncer, hemodiálise crônica. }\end{array}$ \\
\hline $\begin{array}{l}\text { Moderado } \\
\text { (Pontuação 2) }\end{array}$ & $\begin{array}{l}\text { Perda de peso > } 5 \% \text { em } 2 \text { meses ou; } \\
\text { IMC } 18,5-20,5+\text { condição geral comprometida ou; } \\
\text { Ingestão alimentar entre } 25-60 \% \text { da necessidade } \\
\text { normal na semana anterior. }\end{array}$ & $\begin{array}{l}\text { Moderado } \\
\text { (Pontuação 2) }\end{array}$ & $\begin{array}{l}\text { Cirurgia abdominal de grande porte; } \\
\text { acidente vascular cerebral (AVC); } \\
\text { pneumonia grave; } \\
\text { doenças malignas hematológicas (leucemias e } \\
\text { linfomas). }\end{array}$ \\
\hline $\begin{array}{l}\text { Grave } \\
\text { (Pontuação 3) }\end{array}$ & $\begin{array}{l}\text { Perda de peso }>5 \% \text { em } 1 \text { mês ( }>15 \% \text { em } \\
3 \text { meses) ou; } \\
\text { IMC }<18,5+\text { condição geral comprometida ou; } \\
\text { Ingestão alimentar entre } 0-25 \% \text { da necessidade } \\
\text { normal na semana anterior. }\end{array}$ & $\begin{array}{l}\text { Grave } \\
\text { (Pontuação 3) }\end{array}$ & $\begin{array}{l}\text { Trauma craniano; } \\
\text { Transplante de medula óssea; } \\
\text { Pacientes em cuidados intensivos } \\
\text { (APACHE >10) }\end{array}$ \\
\hline \multicolumn{4}{|c|}{$\begin{array}{l}\text { Escore total }= \\
\text { Para calcular o escore total: A. Encontre o escore (de } 0 \text { a 3) para o estado nutricional e para a gravidade da doença (escolher apenas a variável de maio } \\
\text { gravidade); B. Some os dois escores para obter o escore total; C. Se o paciente apresentar idade } \geq 70 \text { anos, adicione } 1 \text { ponto ao escore total para ajustar } \\
\text { fragilidade dos idosos. }\end{array}$} \\
\hline \multicolumn{4}{|c|}{$\begin{array}{l}\text { Pontuação } \geq 3 \text { : o paciente está em risco nutricional e a terapia nutricional deve ser inicada. } \\
\text { Pontuação }<3 \text { : no momento, o paciente não apresenta risco nutricional e deve ser reavaliado semanalmente. Porém, se o paciente tiver indicação de } \\
\text { cirurgia de grande porte, deve-se considerar terapia nutricional para evitar riscos associados. }\end{array}$} \\
\hline
\end{tabular}




\section{RESULTADOS}

Participaram deste estudo 386 pacientes, entre os quais $198(51,3 \%)$ eram mulheres e $188(48,7 \%)$ homens. A média da idade foi $56,5 \pm 20,7$ anos, do peso $74,7 \pm 20,7 \mathrm{~kg}$, da altura $166,4 \pm 13,1 \mathrm{~cm} \mathrm{e}$ do IMC $27,1 \pm 9,0 \mathrm{~kg} / \mathrm{m}^{2}$. A aferição das medidas antropométricas de peso e altura foi realizada em 174 indivíduos, (45,1\% da amostra); nos casos restantes, utilizaram-se os valores autorreferidos pelo paciente e/ou familiar.

Quanto ao risco nutricional, 57 (14,8\%) pacientes tiveram o escore $\geq 3$, caracterizando risco nutricional, e $329(85,2 \%)$ apresentaram o escore $<3$ (Tabela 1).

Não houve diferença entre os gêneros no que se refere ao risco nutricional, sendo observada prevalência deste desfecho em $30(14,6 \%)$ das mulheres e $28(14,9 \%)$ dos homens.

$\mathrm{Na}$ primeira parte da triagem nutricional, a qual é composta por quatro perguntas cujas respostas afirmativas caracterizam risco nutricional, 315 pacientes $(81,6 \%)$ tiveram pelo menos uma resposta afirmativa, sendo a redução de ingestão alimentar na última semana a mais prevalente, verificada em 42 pacientes $(73,7 \%)$, seguida da perda de peso nos últimos três meses, identificada em 40 pacientes (70,2\%). Pacientes em risco nutricional apresentaram proporções significativamente maiores em todas as variáveis relacionadas ao escore de risco (Tabela 2).

Tabela 1. Características gerais da amostra de indivíduos adultos e idosos internados em um hospital da cidade de Farroupilha, RS, Brasil, no período de maio a julho de 2013 $(n=386)$

\begin{tabular}{|c|c|}
\hline Variáveis & Dados \\
\hline \multicolumn{2}{|l|}{ Gênero } \\
\hline Feminino $-\mathrm{n}(\%)$ & $198(51,3)$ \\
\hline Masculino - n(\%) & $188(48,7)$ \\
\hline Idade (anos) - média $\pm D P$ & $56,5 \pm 20,7$ \\
\hline Peso $(k g)$ - média $\pm D P$ & $74,7 \pm 17,2$ \\
\hline Altura $(\mathrm{cm})$ - média \pm DP & $166,4 \pm 13,1$ \\
\hline Índice de massa corporal $\left(\mathrm{kg} / \mathrm{m}^{2}\right)-$ média $\pm D P$ & $27,1 \pm 9,0$ \\
\hline \multicolumn{2}{|l|}{ Risco Nutricional - n(\%) } \\
\hline $\operatorname{Sim}(\geq 3)$ & $57(14,8)$ \\
\hline Não $(<3)$ & $329(85,2)$ \\
\hline
\end{tabular}

Tabela 2. Distribuição dos escores de risco nutricional de acordo com diagnóstico de risco nutricional, de indivíduos adultos e idosos internados em um hospital da cidade de Farroupilha, RS, Brasil, $2013(n=386)$. Escores conforme o protocolo Nutrition Risk Screening (NRS 2002) ${ }^{10}$

\begin{tabular}{|c|c|c|c|c|}
\hline \multirow[b]{2}{*}{ Variáveis } & \multirow{2}{*}{$\begin{array}{l}\text { Amostra total } \\
(n=386) \\
n(\%)\end{array}$} & \multicolumn{2}{|c|}{ Risco Nutricional } & \multirow[b]{2}{*}{$\mathbf{p}$} \\
\hline & & $\begin{array}{c}\operatorname{Sim}(n=57) \\
n(\%)\end{array}$ & $\begin{array}{c}\text { Não }(n=329) \\
n(\%)\end{array}$ & \\
\hline \multicolumn{5}{|l|}{ Parte 1} \\
\hline$I M C<20,5$ & $39(10,2)$ & $20(35,7)$ & $19(5,8)$ & $<0,001^{*}$ \\
\hline PP $3 m$ & $107(27,7)$ & $40(70,2)$ & $67(20,4)$ & $<0,001^{*}$ \\
\hline Redução na ingestão alimentar na última semana & $136(35,2)$ & $42(73,7)$ & $94(28,6)$ & $<0,001^{*}$ \\
\hline Doença grave, está em mau estado geral ou em UTI & $33(8,5)$ & $16(28,1)$ & $17(5,2)$ & $<0,001+$ \\
\hline \multicolumn{5}{|l|}{ Parte 2} \\
\hline Estado Nutricional & & & & $<0,001^{*}$ \\
\hline Ausente & $291(75,4)$ & $8(14,0)$ & $283(86,0)$ & \\
\hline Leve & $53(13,7)$ & $11(19,3)$ & $42(12,8)$ & \\
\hline Moderado & $21(5,4)$ & $17(29,8)$ & $4(1,2)$ & \\
\hline Grave & $21(5,4)$ & $21(36,8)$ & $0(0,0)$ & \\
\hline Gravidade da doença & & & & $<0,001^{*}$ \\
\hline Ausente & $284(73,6)$ & $14(24,6)$ & $270(82,1)$ & \\
\hline Leve & $83(21,5)$ & $29(50,9)$ & $54(16,4)$ & \\
\hline Moderado & $12(3,1)$ & $7(12,3)$ & $5(1,5)$ & \\
\hline Grave & $7(1,8)$ & $7(12,3)$ & $0(0,0)$ & \\
\hline \multicolumn{5}{|l|}{ Pontuação Adicional } \\
\hline Idade $\geq 70$ anos & $102(26,4)$ & $35(61,4)$ & $67(20,4)$ & $<0,001^{*}$ \\
\hline
\end{tabular}

* Teste qui-quadrado de Pearson; + teste exato de Fisher.

IMC: índice de massa corporal; UTI: unidade de tratamento intensivo. 


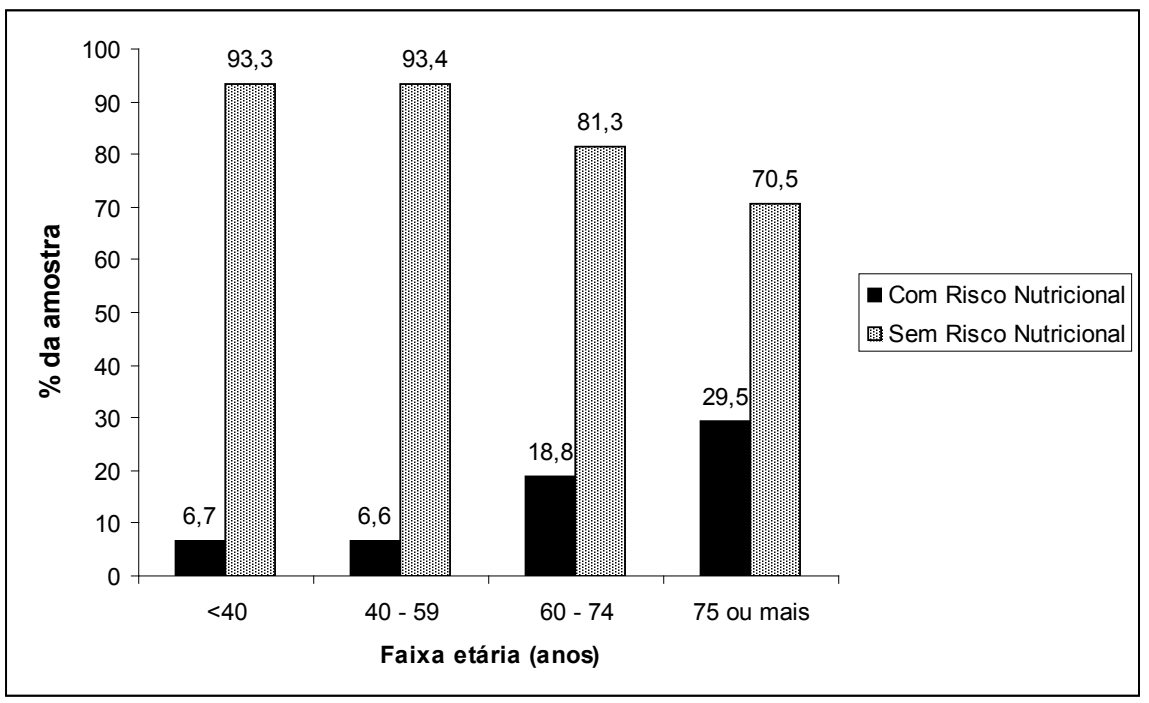

Figura 1. Risco nutricional por quartil de idade de indivíduos adultos e idosos internados em um Hospital da cidade de Farroupilha, RS, Brasil, 2013 ( $n=384)$

$\mathrm{Na}$ segunda parte do instrumento de triagem, que pontua o estado nutricional e a gravidade da doença, observou-se que, em relação ao estado nutricional, a maior proporção foi representada pelo estado grave (21 pacientes, $36,8 \%$ ) seguida do moderado (17 pacientes, $29,8 \%$ ). Quanto à gravidade da doença, prevaleceu o fator de risco leve 29 (50,9\%). A pontuação adicional através da variável idade ( $\geq 70$ anos) contribuiu em 35 pacientes $(61,4 \%)$ para caracterizar o indivíduo em risco nutricional (Tabela 2).

Houve associação entre faixa etária e risco nutricional $(p<0,001)$. A prevalência de risco nutricional aumentou consideravelmente após os 60 anos e cresceu ainda mais na faixa de 75 anos ou mais (Figura 1).

\section{DISCUSSÃO}

O presente estudo verificou a prevalência do risco nutricional nos pacientes em até 72 horas da admissão hospitalar, através do protocolo de triagem de risco nutricional NRS 2002. O risco nutricional foi encontrado em $14,8 \%$ dos pacientes, resultado semelhante ao estudo que incluiu 608 pacientes internados em um departamento de gastroenterologia na Suíça, dos quais $14 \%$ tiveram pontuação $\geq 3$, no ano de $2008,{ }^{18}$ e a um estudo feito em 2009 na Turquia, ${ }^{19}$ em 34 hospitais de diferentes cidades, no qual 15\% dos pacientes avaliados estavam em risco nutricional. É semelhante, também, ao resultado encontrado em estudo realizado na Romênia, com 3.198 pacientes na mesma faixa etária, em que o índice de risco foi de $17,1 \%,{ }^{20} \mathrm{e}$ também ao estudo feito na Croácia no ano de
2010, onde 1.696 pacientes foram avaliados, e destes, $19,3 \%$ estavam em risco nutricional. ${ }^{21}$

Percentuais de risco maiores também são descritos na literatura, como o de um estudo realizado com 1.144 pacientes da rede pública de saúde de Portugal em 2008, ${ }^{22}$ que encontrou $36 \%$ dos pacientes em risco nutricional na admissão hospitalar. Achados também confirmados em um hospital universitário do nordeste brasileiro, em que 99 pacientes foram avaliados na admissão hospitalar no ano de 2010 , e o risco nutricional foi identificado em $39,4 \%$ dos mesmos. Nesse mesmo estudo a baixa ingestão de alimentos foi associada ao risco..$^{23}$

Trabalho realizado em um hospital base em Israel, em 2012, mostrou que dos 504 pacientes avaliados na admissão hospitalar, 159 (31,5\%) estavam com alto risco nutricional. Nesse mesmo estudo, que também analisou a primeira etapa da NRS 2002, observouse que $70 \%$ dos pacientes tiveram pelo menos uma resposta positiva, ${ }^{17}$ enquanto no presente estudo o percentual de pacientes com pelo menos uma resposta positiva na primeira etapa da triagem foi de $81,6 \%$.

No presente estudo, dados referentes à segunda etapa da triagem, a qual avalia os fatores estado nutricional e gravidade da doença, demonstraram que, para o fator estado nutricional, $36,8 \%$ dos indivíduos foram classificados como graves. Quanto à gravidade da doença, prevaleceu o fator de risco leve 50,9\%. Outros estudos mostram, que, dentre as patologias que integram o risco leve, as neoplasias contribuem significativamente para classificar os pacientes em risco nutricional. ${ }^{24-26}$ 
Em ambientes hospitalares, a redução na ingestão alimentar, a perda de peso e a idade estão entre os principais fatores que contribuem para o desenvolvimento de desnutrição. ${ }^{26-28}$ Estudo realizado em 2011 para avaliar a aceitação de dietas de pacientes internados em um hospital público no sul do país mostrou que o percentual de insatisfação dos indivíduos varia de 30 a $50 \%$, dependendo da restrição da dieta. ${ }^{27}$ Pela faixa etária da amostra, com uma grande proporção de pacientes acima dos setenta anos, a pontuação adicional pela idade colaborou em $61,4 \%$ dos casos para caracterizar o indivíduo em risco nutricional, corroborando com outros estudos realizados em diferentes países. ${ }^{3,25,26,29,30}$
Uma das limitações do método utilizado é que depende das respostas do paciente, o qual se encontra no hospital para tratar de algum dano à sua saúde. Nessa situação, o paciente apresenta-se indisposto para o diálogo com os profissionais. Uma limitação específica deste estudo consistiu no número expressivo de casos em que os dados de peso, altura e IMC tiveram que ser autorreferidos devido à impossibilidade de avaliar o paciente fora do leito.

Concluindo, observou-se elevada proporção de indivíduos em risco nutricional, sendo a redução na ingestão alimentar na última semana a variável que mais contribuiu para dar sequência à segunda etapa do processo de triagem nutricional.

\section{REFERÊNCIAS}

1. Stratton RJ, Hackston A, Longmore D, Dixon R, Price S, Stroud M, King C, Elia M. Malnutrition in hospital outpatients and inpatients: prevalence, concurrent validity and ease of use of the "malnutrition universal screening tool" (MUST) for adults. Br J Nutr. 2004;92(5): 799-808.

2. Waitzberg DL, Caiffa WT, Correia M. Hospital Nutrition: The Brazilian National Survey (IBRANUTRI): a Study of 4000 Patients. Nutrition. 2001;17(7-8):573-80.

3. Norman K, Fichard C, Lochs H, Pirlich M. Prognostic impact of disease-related malnutrition. Clin Nutr. 2008;27:5-15.

4. Micheli ET, Abrahão CL, Grigoletti SS, Berizzi V, Cruz LB. Diagnóstico Nutricional: comparação entre os instrumentos de avaliação Nutrition Risk Screening (NRS-2002) e Avaliação Nutricional do Hospital de Clínicas de Porto Alegre. Revista HCPA Porto Alegre. 2009;29(1):23-28.

5. Fontoura CSM, Cruz OD, Londero GL, Vieira MR. Avaliação Nutricional de Paciente Critico. Rev Bras Ter Intensiva. 2006;18(3):298-306.

6. Sampaio RM, Vasconcelos CM, Pinto FJ. Prevalência de desnutrição segundo a avaliação nutricional subjetiva global em pacientes internados em um hospital público de Fortaleza (CE). RBSP. 2010;34(2):311-20.

7. Sociedade Brasileira de Nutrição Parenteral e Enteral - Associação Brasileira de Nutrologia. Triagem e avaliação do estado Nutricional. Projeto Diretrizes. 2011;9(3);1-16.

8. Marcadenti A, Vencatto C, Boucinha, ME, et al. Desnutrição, tempo de internação e mortalidade em um hospital geral do sul do Brasil. Ciênc Saúde. 2011;4(1):7-13.

9. Johansen N, Kondrup J, Plum LM, Bak L, Nørregaard P, Bunch E, Baernthsen H, Andersen JR, Larsen IH, Martinsen A. Effect of nutritional support on clinical outcome in patients at nutritional risk. Clin Nutr. 2004;23(4):539-50.

10. Kondrup J, Allison SP, Elia M, Vellas B, Plauth M. Educational and Clínical Practice committee, European Society for Parenteral and Enteral Nutrition (ESPEN). ESPEN guidelines for nutrition screening 2002. Clin Nutr. 2003;22(4):415-21.

11. Kyle UG, Kossovsky MP, Karsegard VL, Pichard C. Comparison of tools for nutritional assessment and sceening at hospital admission: A population study. Clin Nutr. 2006;25:409-17.

12. Raslan M, Gonzalez MC, Dias MC, Paes-Barbosa FC, Cecconello I, Waitzberg DL. Aplicabilidade dos métodos de triagem nutricional no paciente hospitalizado. Rev Nutr. 2008;21(5):553-61.

13. Rasmussen HH, Holst M, Kondrup J. Measuring nutrition risk in hospitals. Clin Epidemiol. 2009;2:209-16.

14. Elia M, Zellipour L, Stratton RJ. To screen or not to screen for adult malnutrition? Clin Nutr. 2005;24(6):867-84.

15. Bastos NM, Fontoura CSM, Abrahão CLO, Micheli ET, Padilha LM, Simon MI, Maraschin T. Diagnóstico nutricional: uma nova perspectiva na assistência ao paciente adulto do Hospital de Clínicas de Porto Alegre. Rev Bras Nutr Clin. 2008;23(4):282-5.

16. Kondrup J, Johansen N, Plum LM, Bak L, Larsen IH, Martinsen A, Andersen JR, Baernthsen H, Bunch E, Lauesen N. Incidence of nutritional risk and causes of inadequate nutritional care in hospitals. Clin Nutr. 2002;21(6):461-8.

17. Giryes S, Leibovitz E, Matas Z, Fridman S, Gavish D, Shalev B, Ziv-Nir Z, Berlovitz Y, Boaz M. Measuring Nutrition risk in hospitalized patients: MENU, a hospital-based prevalence survey. IMAJ. 2012;14(7):405-9.

18. Schiesser M, Müller S, Kirchhoff P, Breitenstein S, Schäfer M, Clavien PA. Assessment of a novel screening score for nutritional risk in predicting complications in gastro-intestinal surgery. Clin Nutr. 2008;27(4):565-70.

19. Korfali G, Gündoğdu H, Aydintuğ S, Bahar M, Besler T, Moral AR, Oğuz M, Sakarya M, Uyar M, Kiliçturgay S. Nutritional risk of hospitalized patients in Turkey. Clin Nutr 2009:28(5):533-7. 
20. Gheorghe C, Pascu O, Iacob R, Vadan R, Iacob S, Goldis A, Tantau M, Dumitru E, Dobru D, Miutescu E, Saftoiu A, Fraticiu A, Tomescu $\mathrm{D}$, Gheorghe L. Nutritional risk screening and prevalence of malnutrition on admission to gastroenterology departments: a multicentric study. Chirurgia (Bucur). 2013(4);108:535-41.

21. Pavic T, Ljubicic N, Stojsavljevic S, Krznaric Z. Nutritional Screening Model in Tertiary Medical Unit in Croatia. Ann Clin Nutr. 2012;61(1):65-9.

22. Amaral TF, Matos LC, Teixeira MA, MM Tavares, Alvares L, Antunes A. Undernutrition and associated factors among hospitalized patients. Clin Nutr. 2010;29(5):580-5.

23. Silva LD, Santos AP, Cabral CP, Burgos APGM. A triagem nutricional em pacientes clínicos em um hospital universitário no Nordeste do Brasil. Nutr Hosp. 2012;27(2):469-76.

24. Haldun G, Eren E, Recep A, Hakan K, Mehmet O, Vedat O, Tanju A. Evaluation of nutritional risk on admission to the general surgery department. Bratisl Lek Listy. 2008;109(2):57-60.

25. Vandewoude MF, Alish CJ, Sauer AC, Hegazi RA. Malnutrition-Sarcopenia Syndrome: Is This the Future of Nutrition Screening and Assessment for Older Adults? J Aging Res. 2012;2012(1):1-8.

26. Pirlich M, Schutz T, Norman K, et al. The German hospital malnutrition study. Clin Nutr. 2006;25(4):563-72.

27. Sousa AA, Gloria MS, Cardoso TS. Aceitação de dietas em ambiente hospitalar. Rev Nutr. 2011:24(2):287-94.

28. Aquino RC, Philippi ST. Identificação de fatores de risco de desnutrição em pacientes internados. Rev Assoc Med Bras. 2011:57(6):637-43.

29. Burgos R, Sarto B, Elío I, Planas M, Forga M, Cantón A, Trallero R, Muñoz MJ, Pérez D, Bonada A, Saló E, Lecha M, Enrich G, Salas-Salvadó J. Prevalência de malnutrición y sus factores etiológicos en hospitales. Nutr Hosp. 2012;27(2):469-76.

30. Neelemaat F, Bosmans JE, Thijs A, Seidell JC, Marian AE. Oral nutritional support in malnourished elderly decreases functional limitations with no extra costs. Clin Nutr. 2012;31:183-90. 